International Journal of Distributed and Parallel systems (IJDPS) Vol.1, No.1, September 2010

\title{
A BANDWIDTH EFFICIENT SCHEDULING FRAMEWORK FOR NON REAL TIME APPLICATIONS IN WIRELESS NETWORKS
}

\author{
G.Indumathi 1 and K.Murugesan2 \\ 1Department of Electronics and Communication Engineering, Mepco Schlenk Engineering \\ College, Anna University, Chennai, India \\ indupriyanga@gmail.com \\ 2Barathiyar Institute of Engineering for Women, Attur, Anna University, Chennai, India
}

\begin{abstract}
The key concern on the bandwidth allocation and scheduling for non real time traffic are the fulfillment of its minimum throughput requirement and improvement of bandwidth utilization with acceptable delay. This paper proposes a simple and efficient scheduling framework for allocating bandwidth to non real time polling service (nrtPS) users in IEEE 802.16 networks. In this framework, jointly selective repeat ARQ at the MAC layer and adaptive modulation and coding techniques at the physical are considered. Numerical simulations demonstrate that the proposed scheduling approach provides a graceful compromise between bandwidth utilization and packet delivery delay while maintaining the minimum throughput requirements of nrtPS applications. Thus proves the efficiency of the proposed framework. The simulation is done for unicast scenario.
\end{abstract}

\section{KEYWORDS}

Adaptive Modulation and Coding (AMC), Automatic Repeat request (ARQ), Scheduling, IEEE 802.16 networks, Markov models.

\section{INTRODUCTION}

The rapid growth in demand for high-speed and high quality multimedia communications is creating opportunities and challenges for next-generation wired-wireless network designs. Multimedia communications entail diverse quality of service (QoS) requirements for different applications including voice, data and real time, or streaming video/audio. Providing QoS-guaranteed services is necessary for future wireless networks, including cellular networks, mobile ad hoc networks, and wireless sensor networks, e.g., IEEE 802.16, IEEE 802.11, and IEEE 802.15 standard wireless networks. Such networks are envisioned to support multimedia services with different QoS requirements. However, the aforementioned standards define only QoS architecture and signaling, but do not specify the scheduling algorithm that will ultimately provide QoS support.

Four QoS services are defined by IEEE 802.16 standard:

1) Unsolicited grant service (UGS) supports constant bit rate (CBR) or fixed throughput connections such as E1/T1 lines and voice over IP (VoIP). This service provides guarantees on throughput, latency, and jitter to the necessary levels as TDM services. The QoS metrics here are

the packet error rate (PER) and the service rate. 
2) Real-time polling service (rtPS) provides guarantees on throughput and latency, but with greater tolerance on latency relative to UGS, e.g., MPEG video conferencing and video streaming. The delayed packets are useless and will be dropped. The QoS metrics are the PER and the maximum delay (or the maximum delay for a given outage probability).

3) Nonreal-time polling service (nrtPS) provides guarantees in terms of throughput only and is therefore suitable for mission critical data applications, such as File Transfer Protocol (FTP). These applications are time-insensitive and require minimum throughput. For example, an FTP

file can be downloaded within a bounded waiting time if the minimum reserved rate is guaranteed. The QoS metrics are the PER and the minimum reserved rate.

4) Best effort (BE) service provides no guarantees on delay or throughput and is used for Hypertext Transport Protocol (HTTP) and electronic mail (e-mail), for example. BE applications receive the residual bandwidth after the bandwidth is allocated to the connections of the previous three service classes. Although no delay and rate is specified for BE connections, a prescribed PER should be maintained over wireless channels.

The signaling and procedure for the service setup and maintenance of each connection are defined as in the IEEE 802.16 standard [1]. However, the standard does not define the scheduling

mechanism or the admission control and traffic policing processes. The signaling overhead is not included in our design and analysis.

Many resource allocation and scheduling schemes have been developed to deal with the four service types based on their intrinsic characteristics and QoS requirements. For UGS, a common solution is to periodically grant a fixed amount of resources since the service is designed for the CBR applications. For the rtPS applications, scheduling schemes, such as the largest weighted delay first and strict priority scheduling, were proposed to deal with the stringent delay requirement [2],[3]. For BE service, proportional fairness scheduling is an effective approach to balance the system throughput and fairness. To the best of our knowledge, very few research efforts have dedicatedly dealt with the resource allocation and scheduling for nrtPS in spite of its ultimate importance. It has been reported that transmission control protocol (TCP) traffic (which exclusively takes nrtPS as the carrier in an IEEE 802.16 network) may take up to $80 \%$ of the total Internet bandwidth [4], while an average share of $83 \%$ is observed in the High-Performance Computing (HPC) networks. With such predominant bandwidth consumption, it is crucial to develop a dedicated strategy for dealing with nrtPS traffic in IEEE 802.16 networks. The key concerns on the resource allocation and scheduling for nrtPS traffic are the fulfillment of its minimum throughput requirement and improvement of resource utilization with

acceptable delay. Efforts on improving resource utilization and reducing experienced delay are in general contradictory since high resource utilization can be achieved by assigning the resource to the subscriber stations (SSs) with good channel conditions, while leaving the SSs with poor channel conditions starved and experienced a long delay. Although nrtPS connections are delay-tolerant, they should not be starved for too long since otherwise the flows will suffer considerable performance degradation. Therefore, 
how to compromise the resource utilization and experienced delay of each SS is a challenging and important issue.

In this paper an efficient resource allocation and packet scheduling frame work is proposed for nrtPS traffic in IEEE 802.16 networks. An analytical model is used to investigate some important performance measures like inter service time, PDU delivery delay, good put and spectral efficiency. The analytical model characterizes the Adaptive Modulation and Coding at the Physical layer and elective automatic repeat request mechanism at the data link layer. Simulation results are given to verify the analytical model and to demonstrate the proposed scheduling frame work efficiency.

The rest of the paper is organized as follows: Section II introduces the associated work on the scheduling and ARQ mechanism. Section III describes the proposed resource allocation and scheduling frame work. Section IV corresponds to the numerical results, which verifies the efficiency of the frame work and the accuracy of analytical model. Finally concluding remarks are given in Section V.

\section{ASSOCIATED WORK ON THE SCHEDULING AND ARQ MECHANISM}

Many wireless scheduling algorithms have been designed to support data traffic in $2 \mathrm{G} / 3 \mathrm{G}$ wireless systems. Most of them focused on either real time applications with stringent delay or Best Effort application with improved throughput and fairness. Opportunistic scheduling [5] was devised to maximize the resource utilization by assigning resources to the user with the best channel condition at each timeslot. Nonetheless, it is not suitable for nrtPS applications since long-term starvation could occur for the SSs with bad channel conditions. An opportunistic fair scheduling was proposed in [6] to maintain the long-term fairness and achieve a smooth service rate. The fore mentioned schemes focused on improving fairness performance, rather than providing the guarantee for the minimum throughput requirement, which is essential for non real time traffic.

The work in [7] assigns priorities to different traffics to satisfy their QoS requirements, but it does not consider the fairness issue. The work in [8] models the scheduling problem as an $\mathrm{M} / \mathrm{M} / 1 / \mathrm{K}$ queuing system, whose objective is to minimize the blocking probability. However, it may not guarantee the delays of real time traffics. In [9], authors proposed a fair queuing algorithm as the bandwidth allocation policy and multicode CDMA as the physical layer transmission and retransmission technique to analyze the problem of queing delay in wireless networks. Also investigates how information about channel quality can be incorporated into the scheduler. This work considers the delay requirements and not the throughput or resource utilization. So it is not suitable for nrtPS applications. Adaptive EXP/PF scheduling in [10] focused on BE traffic and real-time service, where the service priority was evaluated based on the experienced delay and channel condition at each queue.

A few studies have considered non-real-time applications, but they only focused on satisfying either the throughput ratio or the acceptable delay [11]-[13]. The authors in [11] propose a modified proportional fair (MPF) method to increase the network throughput while maintaining fairness. In [12], a proportional fairness scheme based on signal-to-noise ratio is proposed to achieve rate maximization is proposed. However, the above studies consider only non-real-time traffics. The Channel-Aware Round Robin 
(CARR) scheme [13] provided a soft bandwidth guarantee by compromising the conventional round robin and opportunistic scheduling. However, this scheme fails to guarantee the minimum bandwidth since the allocated resource is entirely determined by the corresponding long-term channel condition of each end user instead of the bandwidth demand.

Automatic Repeat reQuest (ARQ) is an important closed-loop error control mechanism to achieve a low packet loss rate in error prone wireless environments. Although ARQ schemes have been extensively studied, some new challenges have emerged with the launching of the IEEE 802.16 standard. The IEEE 802.16 standard specifies some advances in physical layer techniques and media access control protocol, such as AMC, orthogonal frequency division multiplexing (OFDM), flexible retransmission of lost packets, etc. These characteristics have creates fundamental differences in the efforts of performance analysis of ARQ in IEEE 802.16 networks compared with that in many previous works. The assumption of constant packet transmission time in the previous work does not hold in IEEE 802.16 networks due to the adoption of AMC. With AMC, the link capacity varies with the channel conditions, leading to the fact that the time taken to retransmit a packet varies with the current channel condition, and may be different from that taken in the previous transmission attempt. Also, most of the former studies commonly assumed that the lost packets are retransmitted immediately in the very next timeslot [14]-[17]. This is a very strong assumption because the time consumed by data decoding, processing, and feedback is not negligible when the round-trip time is considered. In addition, the assumption may result in low system capacity due to varying channel conditions. For instance, when the channel condition of the next timeslot is poor, the retransmission could experience a much higher packet loss rate. In IEEE 802.16 networks, the retransmission of lost packets is flexible and does not just occur within the very next timeslot. Therefore, a fundamental difference can be identified in the design and performance analysis in IEEE 802.16 networks compared with that in many previous works. In [19], a low complexity sub optimal algorithm based on a cross-layer approach that combines information provided by the PHY-MAC layers is discussed. In [20], jointly optimized power allocation and channel coding to improve the quality of images and video sequences when transmitted over wireless channels are discussed. Thus, it is critical to develop an efficient resource allocation and scheduling framework by jointly considering the selective ARQ mechanism at the media access control (MAC) layer and AMC technique at the physical layer that not only can maintain the minimum bandwidth requirements for nrtPS flows, but also initiate a graceful compromise between the resource utilization and delivery delay.

\section{III.THE PROPOSED RESOURCE ALLOCATION AND SCHEDULING FRAME WORK}

In the proposed resource allocation and scheduling frame work for nrtPS traffic in IEEE 802.16 networks the following steps are taken:

i) AMC technique is considered at the physical layer as specified in IEEE 802.16 standard, where modulation levels are dynamically adjusted to impose the channel 
condition of each subscriber station (SS). Different modulation levels lead to different numbers of information bits carried by an OFDM symbol.

ii) Selective ARQ mechanism is considered at the MAC layer. Using this, the information about whether or not protocol data units (PDUs) transmitted at a downlink sub frame are successfully received is feedback to the base station at the uplink sub frame. Based on that, the failed PDUs are retransmitted next time when the queue obtains the transmission opportunity, instead immediately. By taking the impact of selective ARQ on the retransmission of lost PDUs, the performance of the proposed scheduling frame work is analyzed in terms of achieved goodput and the packet deliverance delay for each SS.

iii) Two operational parameters are considered. Let them be $l$ and $B$. $l$ is the number of SSs selected at each MAC frame, controls the tradeoff between the resource utilization and the packet deliverance delay of each SS; $B$ is the bandwidth granted to a SS when it is served, which fully satisfies the bandwidth requirement of nrtPS traffic.

iv) The channel condition of each SS is taken into account in terms of signal to noise ratio.

The proposed scheduling frame work works as follows. At the beginning of each MAC frame, $l$ SSs with good channel conditions are selected and granted with transmission opportunities at the down link sub frame. Each selected SS is assigned with a specific amount of resources, denoted by $B$ according to the minimum throughput requirement of nrtPS traffic destined to the particular SSs and the channel conditions of all SSs.

An analytical model is considered to evaluate the performance metrics by including impact of two performance parameters $l$ and $B$ on the design requirements like resource utilization, minimum bandwidth requirement and packet deliverance delay. Some key performance metrics considered are inter-service time, PDU deliverance delay, goodput, resource utilization and spectral efficiency. The following assumptions are made in the performance analysis:

(1) a link layer SDU corresponds to an IP packet;

(2) all PDUs are of equal size with L bits;

(3) feedback information of PDUs transmitted at a downlink sub frame is sent back to the

BS through the uplink sub frame.

all

(4) to support a well defined connection admission control policy in the network, resources available for nrtPS traffic are admitted into the network at each MAC frame.

(5) when a queue is scheduled, it has PDUs waiting for transmission.

\section{A. Wireless Channel model}

A finite state Markov channel (FSMC) model [18] is used to describe the timevarying channel condition of each SS. In addition, when the channel model is constructed, the discrete AMC architecture defined in the IEEE 802.16 standard is taken into account, where the received 
Signal-to-noise ratio (SNR) is divided into several disjoint regions. According to the perceived SNR, the BS selects a proper modulation level and coding scheme for each SS. The boundaries of SNR for the $(N+1)$-state FSMC is denoted as $\left\{\gamma_{n}\right\}_{n=0}^{N+1}$. When the received SNR is located in the $\operatorname{set}\left[\gamma_{n}, \gamma_{n+1}\right)$, the channel state is represented by state $n$. Based on the IEEE 802.16 standard, an 8-states Markov channel model is used in the paper. The values of the modulation and coding levels corresponding to each channel state are specified in Table I.

Table I Modulation and coding levels for IEEE 802.16 networks

\begin{tabular}{|c|c|c|c|}
\hline $\begin{array}{c}\text { State ID } \\
n\end{array}$ & $\begin{array}{c}\text { Modulation level } \\
\text { and Coding }\end{array}$ & $\begin{array}{c}\text { Information } \\
\text { (Bits/OFDM } \\
\text { Symbol) }\end{array}$ & $\gamma_{\mathrm{n}} \mathrm{dB}$ \\
\hline 0 & No transmission & 0 & 0 \\
\hline 1 & BPSK(1/2) & 96 & 3 \\
\hline 2 & QPSK(1/2) & 192 & 6 \\
\hline 3 & QPSK(3/4) & 288 & 8.5 \\
\hline 4 & 16QAM(1/2) & 384 & 11.5 \\
\hline 5 & 16QAM(3/4) & 576 & 15 \\
\hline 6 & 64QAM(2/3) & 768 & 18.5 \\
\hline 7 & 64QAM(3/4) & 864 & 21 \\
\hline
\end{tabular}

The probability of staying at state $n$ is given by [18]

$$
P_{r}(n)=\frac{\Gamma\left(m, m \cdot b_{n} / \bar{\gamma}\right)-\Gamma\left(m, m \cdot b_{n+} \bar{\gamma}\right)}{\Gamma(m)}
$$

where $\bar{\gamma}$ is the average SNR, $m$ is Nkagami fading parameter, $\Gamma(m)$ is the Gamma function and $\Gamma(m, \gamma)$ is the incomplete Gamma function. The channel becomes a Rayleigh fading channel when $m=1$.

Assuming slow fading conditions so that transition happens only between adjacent states, the adjacent-state transition probability can be determined by

$$
\begin{aligned}
& P_{n, n+1}=\frac{N_{n+1} T_{f}}{P_{r}(n)} \text {, if } n=0, \cdots N-1 \\
& P_{n, n+1}=\frac{N_{n} T_{f}}{P_{r}(n)} \quad \text { if } n=1, \cdots, N
\end{aligned}
$$

The probability of staying at the same state $n$ is given by 


$$
P_{n, n}= \begin{cases}1-P_{n, n+1}-P_{n, n-1} & , \text { if } 0<n<N \\ 1-P_{0,1} & \text {, if } n=0 \\ 1-P_{N, N-1} & \text {, if } n=N\end{cases}
$$

where $T_{f}$ is the MAC frame duration and $N_{n}$ is the level cross-rate of state $n$, which can be estimated by

$$
N_{n}=\sqrt{2 \pi \frac{m \gamma_{n}}{\bar{\gamma}}} \frac{f_{d}}{\Gamma(m)}\left(\frac{m \gamma_{n}}{\bar{\gamma}}\right)^{m-1} \exp \left(-\frac{m \gamma_{n}}{\bar{\gamma}}\right)
$$

where $f_{d}$ is the Doppler frequency.

Thus we model the state transition probability matrix as

$$
P_{c}=\left[\begin{array}{cccc}
P_{0,0} & P_{0,1} & \cdots & 0 \\
P_{1,0} & P_{1,1} & P_{1,2} & \vdots \\
0 & \ddots & \ddots & 0 \\
\vdots & P_{N-1, N-2} & P_{N-1, N-1} & P_{N-1, N} \\
0 & \cdots & P_{N, N-1} & P_{N, N}
\end{array}\right]
$$

\section{B. Service Probability for a SS}

The Service Probability is defined as the probability for a SS to obtain the service at a down link sub frame. First, we classify all SSs into three groups denoted as $G_{1}, G_{2}$ and $G_{3}$ based on the channel state of the labeled SS which is under discussion at a specific MAC frame. By giving the channel state of labeled SS as $n$, the three groups of SSs are with channel conditions better than, same as and worse than the state $n$ respectively. Let $k_{1}, k_{2}$ and $k_{3}$ denote the number of $S s$ in the groups $\mathrm{G}_{1}, \mathrm{G}_{2}$ and $\mathrm{G}_{3}$ respectively. For instance, the labeled SS belongs to $\mathrm{G}_{2}$, then the chance of transmission for the labeled SS is only when $k_{1}<l$ holds. Otherwise, all the selected SSs should come from the group $\mathrm{G}_{1}$. When the condition is satisfied, the probability that the labeled SS obtains the chance of transmission can be derived based on the values of $k_{1}$ and $k_{2}$. Since the total number of selected SSs is $l$ and $k_{1}$ SSs are at the channel states better than state $n, l-k_{l}$ is the quota left for the SSs at $\mathrm{G}_{2}$ and $\mathrm{G}_{3}$. When $l-k_{1}$ is larger than or equal to $k_{2}$, all the SSs at $\mathrm{G}_{2}$ are selected. On the other hand, when $k_{2}>l-k_{1}$, the BS randomly selects $l-k_{1}$ out of $k_{2} \mathrm{SSs}$ $\mathrm{u}=$ in the group $\mathrm{G}_{2}$. Therefore the labeled SS obtains the chance of transmission with a probability $\left(l-k_{1}\right) / k_{2}$ which is given as

$$
Q\left(\frac{l-k_{1}}{k_{2}}\right)= \begin{cases}1, & l-k_{1} \geq k_{2} \\ \frac{l-k_{1}}{k_{2}}, & l-k_{1}<k_{2}\end{cases}
$$

\section{Inter Service Time}

An integrated Markov model consists of $2 \mathrm{~N}+1(\mathrm{~N}=6$ in the study) states are considered. These states are grouped as $\mathrm{O}$ and $\mathrm{D}$, where the labeled SS obtains and drops 
the chance of transmission respectively. Let $(n, o)$ and $(n, d)$ be the channel states of labeled SS obtains and drops the chance of transmission respectively.

The transition probabilities of the grouped states are given by

$$
\begin{aligned}
& p_{o d}=\frac{\sum_{n=1}^{7}\left[\theta(n, o) \sum_{j=0}^{7} p_{n o, j d}\right]}{\sum_{n=1}^{7} \theta(n, o)}, \quad p_{o o}=1-p_{n o} \\
& p_{d o}=\frac{\sum_{n=0}^{7}\left[\theta(n, d) \sum_{j=0}^{7} p_{n d, j o}\right]}{\sum_{n=0}^{7} \theta(n, d)}, p_{d d}=1-p_{d o}
\end{aligned}
$$

where $\theta(n, o)$ is the steady state probability of the state $(n, o)$ and $p_{n o, j d}$ is the one step transition probability from the state $(n, o)$ to the state $(j, d)(n=1,2, \ldots 7, j=0,1, \ldots 7)$.

Let $m$ denote the inter service time, which I defined as the duration between two successive transmission chances for the labeled SS. The mean of $m$ is given by

$$
E(m)=\sum_{i=1}^{\infty} i p_{o d}\left(p_{d d}^{i-1} p_{d o}\right)
$$

\section{PERFORMANCE MEASURES}

\section{1) Goodput}

Goodput is defined as the average data rate successfully transmitted by the labeled $\mathrm{SS}$, measured in bps. it is given by

$$
G=\frac{E(\eta) \cdot B}{T_{f}(E(m)+1)}=\frac{L \cdot\left(1-p_{e}\right) \cdot B}{T_{f}(E(m)+1)} \text { bps }
$$

where $\mathrm{E}(\mathrm{m})$ is the mean of the inter service time, $\eta$ is the number of PDUs successfully transmitted by the labeled SS during each transmission opportunity, B is the size of a PDU, $P_{e}$ and $\mathrm{E}(\eta)$ is the mean of $\eta$ calculated by

$$
E(\eta)=\sum_{i=0}^{L}\left[i\left(\begin{array}{l}
L \\
i
\end{array}\right)(1-p)^{i} p^{L-i}\right]=L .(1-p)
$$

2) Delivery Delay of a PDU

The average delivery delay of a PDU is given by

$$
E\left(D_{P D U}\right)=\frac{p_{e}}{1-p_{e}}(E(m)+1)
$$




\section{3) Resource Utilization}

The resource utilization is defined as the number of information bits carried by an OFDM symbol. When a high level modulation I used, the number of information bit carried by an OFDM symbol is large, which gives a high resource utilization and vice versa. It is obtained as

$\operatorname{Re} s . U t i l=\sum_{i=1}^{M}\left[\frac{\sum_{n=1}^{7} \theta_{i}(n, o)}{\sum_{j=1}^{M} \sum_{n=1}^{7} \theta_{j}(n, o)} \sum_{k=1}^{7} I_{k} \frac{a_{k} \theta_{i}(k, o)}{\sum_{n=1}^{7} a_{n} \theta_{i}(n, o)}\right]$

Where $\mathrm{M}$ is the total number of SSs in the network, $\theta_{i}(n, o)$ is th steady state probability of the state $(n, o)$ for $\mathrm{SS}_{i}, \mathrm{I}_{k}$ is the information bits carried by an OFDM symbol when the channel state is at $k$, which I given in Table $\mathrm{I}$ and $\mathrm{a}_{n}$ is the required OFDM symbols to transmit $L$ PDUs at the labeled SS when the channel state is $n$.

\section{4) Spectral Efficiency}

The system spectral efficiency is defined as the maximum throughput / goodput summed over all users in the system divided by the channel bandwidth, measured in $\mathrm{bit} / \mathrm{sec} / \mathrm{Hz}$.

\section{RESULTS AND DISCUSSION}

In the simulation, a Rayleigh fading channel model is adopted. The Downlink/Uplink sub frame duration is fixed as $1 \mathrm{~ms}$ with OFDM symbol duration as 24 $\mu \mathrm{s}$. The channel bandwidth is taken as $5 \mathrm{MHz}$, Doppler frequency $\mathrm{f}_{\mathrm{d}}$ as $15 \mathrm{~Hz}$ and error probability as $10^{-2}$. The parameter to fulfill the satisfaction of the minimum bandwidth requirement of a nrtPS application in terms of PDUs is considered as [ [5 10152025 30]. Fig. 1 shows the impact of the parameter $l$ on the PDU delivery delay for a labeled SS. It can be observed that the PDU delivery delay decreases with the increase of $l$. When the number of PDUs included in a MAC frame at each transmission opportunity increases; the corresponding frame is subject to good channel condition with good SNR, then that PDU experiences a quiet small delivery delay. With the increase of $l$ i.e., the number of subscriber stations in the network increases, the labeled SS is getting more amount of bandwidth, therefore its PDU delivery delay decreases accordingly. 


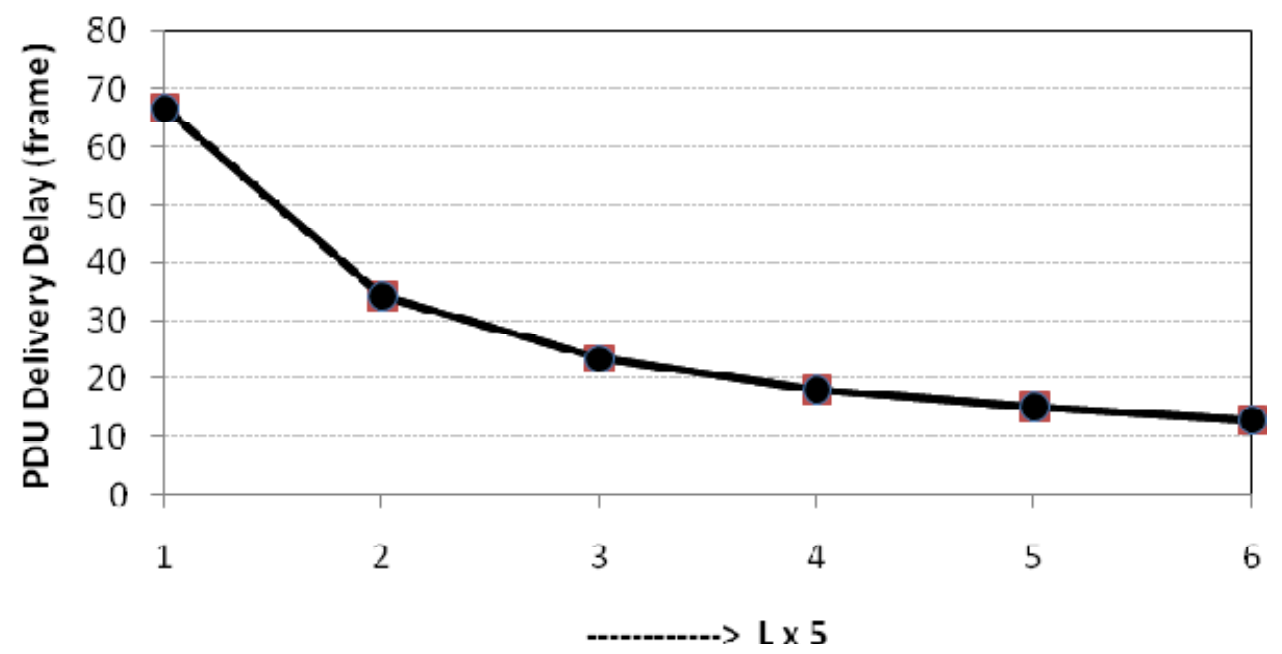

Fig.1 PDU Delivery Delay vs Number of SSs in a network

Fig. 2 shows the impact of the number of SS in a network $l$ on bandwidth utilization. It is observed that the bandwidth utilization increases with the increase of $l$ and the corresponding Signal-to-Noise ratio also. The SNR boundaries for the AMC levels are considered for observation. With poor/worst channel condition with very low SNR, SS can achieve the choice of transmission in a rare situation and so it acquires minimum bandwidth for its PDU transmission. As a result low bandwidth utilization is obtained. At the same time, when the channel is under good condition (high SNR), SS can achieve the best chance of transmissions, therefore a high bandwidth can be utilized by the SS.

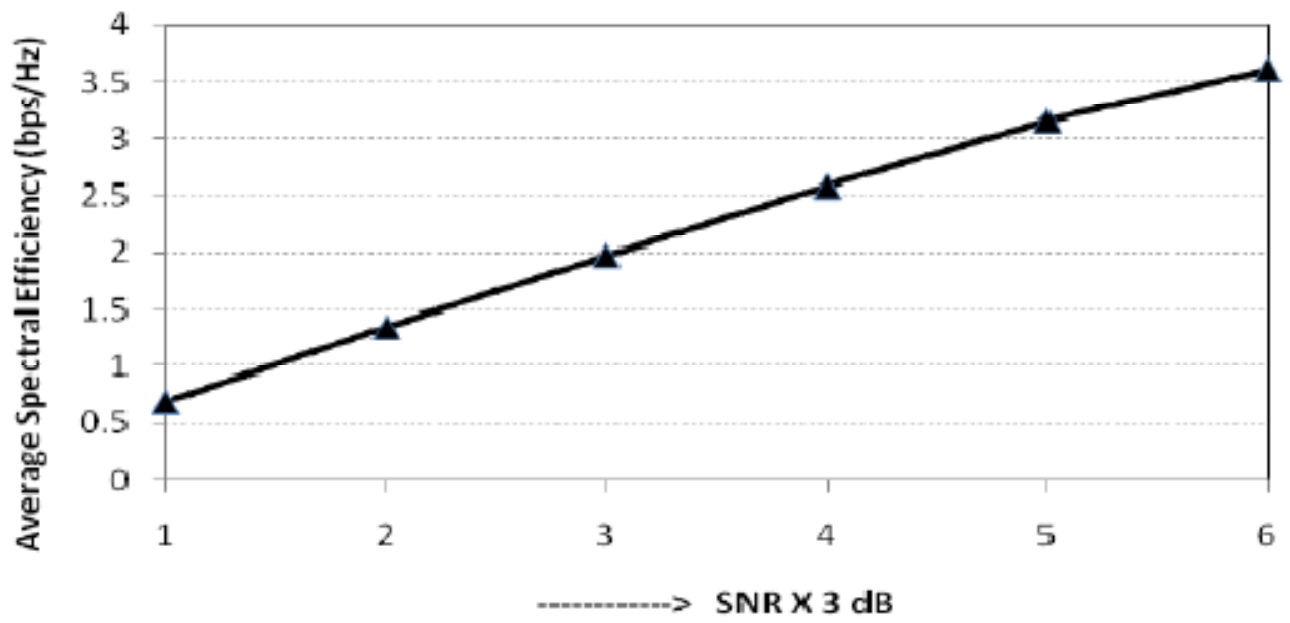

Fig.2 SNR vs Average Spectral Efficiency 


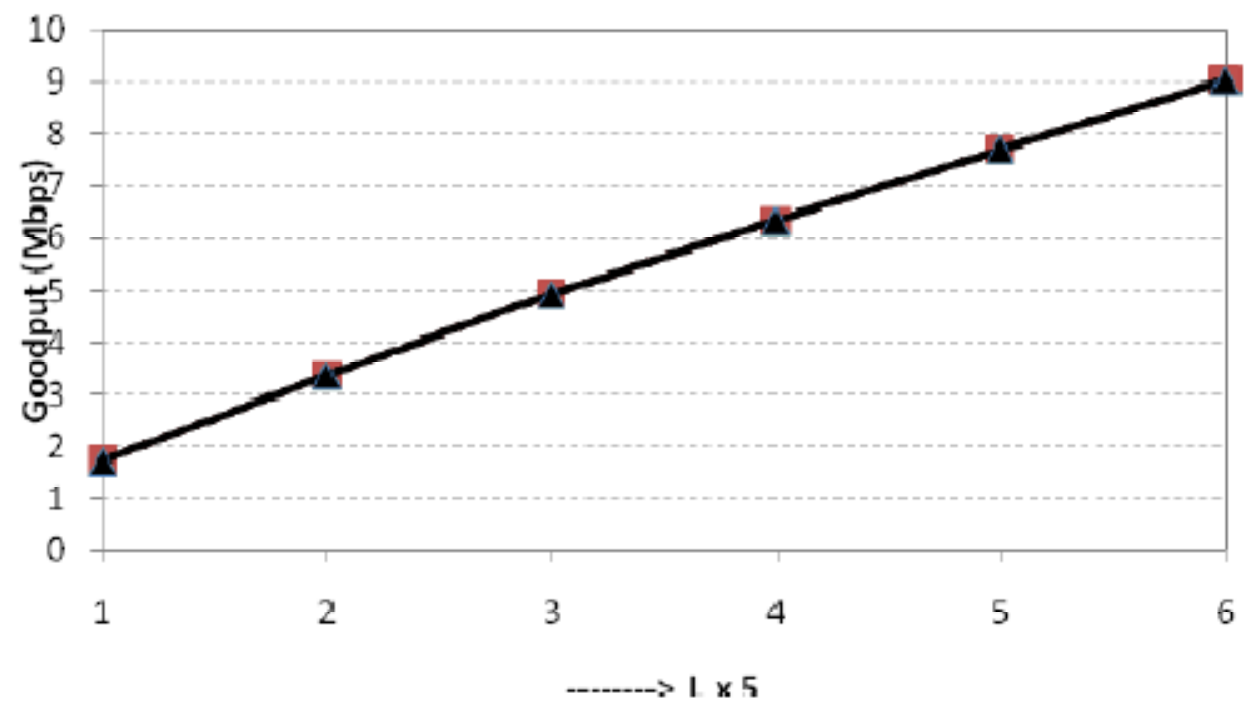

Fig.3 Number of SSs in a network $l$ vs Goodput

Fig.3 shows the relation between the acquired Goodput and the number of SSs $l$ in a network. With the of number of SSs, the achieved goodput of a labeled SS increases accordingly. With the fixed bandwidth grant or with a fixed number of SS in a network, a labeled SS with good channel condition achieves a higher throughput since it can do more number of transmissions.

\section{CONCLUSION}

The proposed bandwidth allocation and scheduling framework is simple and efficient and it is proved for nrtPS applications in IEEE 802.16 environmental setup. In this scheduling framework, AMC technique at the physical layer and selective repeat ARQ at the MAC layer are jointly considered. The developed analytical model for the proposed framework for parameter manipulation is efficiently verified using simulations. The corresponding performance metrics like Goodput, Spectral efficiency and PDU delivery delay are investigated for performance evaluation. This entire framework is done for unicast applications. The same work may be extended for multicast scenario. Also, in this paper, a Nakgami fading distributed eight state Markov channel model is used to focus unicast scheduling for non-real-time polling service applications. In future a MIMO wireless channel model may be considered to optimize the delay performance of packet queuing at the data-link layer to transmit a delay sensitive and bursty real time traffic services.

\section{REFERENCES}

[1] IEEE Standard 802.16 Working Group, IEEE Standard for Local and Metropolitan Area NetworksP art 16: Air Interface for Fixed Broadband Wireless Access Systems (Revision of IEEE Standard 802.16-2001), 2004. 
International Journal of Distributed and Parallel systems (IJDPS) Vol.1, No.1, September 2010

[2] K. Wongthavarawat and A. Ganz, "Packet scheduling for QoS support in IEEE 802.16 broadband wireless access systems," Int. J. Commun. Syst., vol. 16, no. 1, pp. 8196, 2003.

[3] M. Andrews, K. Kumaran, K. Ramanan, A. Stolyar, P. Whiting, and R. Vijayakumar, "Providing quality of service over a shared wireless link," IEEE Commun. Mag., vol. 39, no. 2, pp. 150-154, 2001.

[4] C. Fraleigh, S. Moon, B. Lyles, et al., "Packet-level traffic measurements from the sprint IP backbone," IEEE Network, vol. 17, no. 6, pp. 6-16, 2003.

[5] X. Liu, E. K. P. Chong, and N. B. Shroff, "A framework for opportunistic scheduling in wireless networks," Int. J. Computer and Telecommun. Networking, vol. 41, no. 4, pp. 451-474, 2003.

[6] M. Mehrjoo, M. Dianati, X. Shen, and K. Naik, "Opportunistic fair scheduling for the downlink of IEEE 802.16 wireless metropolitan area networks," in Proc. Qshine'06, Waterloo, Ontario, Canada, Aug. 2006.

[7] E. Hossain and V. K. Bhargava, "Link-level traffic scheduling for providing predictive QoS in wireless multimedia networks," IEEE Trans. Multimedia, vol. 6, no. 1, pp. 199-217, Feb. 2004.

[8] N. A. Ali, M. Hayajneh, and H. Hassanein, "Cross layer scheduling algorithm for IEEE 802.16 broadband wireless networks," in IEEE International Conference on Communications, 2008, pp. 3858-3862.

[9] Anastasios Stamoulis, Nicholas D.Sidripoulis, Georgios B. Giannakis, "Time varying fair queuing scheduling for multi code CDMA based on dynamic programming,"IEEE Trans. On Wireless Communication, vol.3, no.2, March 2004.

[10] J. H. Rhee, J. M. Holtzman, and D. K. Kim, "Performance analysis of the adaptive EXP/PF channel scheduler in an AMC/TDM system," IEEE Commun. Lett., vol. 8, no. 8, pp. 497-499, 2004.

[11] J. Kim, E. Kim, and K. S. Kim, "A new efficient BS scheduler and scheduling algorithm in WiBro systems," in IEEE International Conference on Advanced Communication Technology, vol. 3, 2006, pp. 1467-1470. 
International Journal of Distributed and Parallel systems (IJDPS) Vol.1, No.1, September 2010

[12] Y. Ma, "Rate-maximization scheduling for downlink OFDMA with long term rate proportional fairness," in IEEE International Conference on Communications, 2008, pp. 3480-3484.

[13] H. Wei and R. Izmailov, "Channel-aware soft bandwidth guarantee scheduling for wireless packet access," in Proc. IEEE Wireless Commun. and Networking Conference (WCNC'04), Atlanta, Georgia, Mar. 2004.

[14] J. G. Kim and M. M. Krunz, "Delay analysis of selective repeat ARQ for a Markovian source over a wireless channel," IEEE Trans. Veh. Technol., vol. 49, no. 5, pp. 1968-1981, 2000.

[15] H. Shen, L. Cai, and X. Shen, "Performance analysis of TFRC over wireless links with truncated link level ARQ," IEEE Trans. Wireless Commun., vol. 5, no. 6, pp. 14791487, June 2006.

[16] F. Hou, P. H. Ho, and X. Shen, "A novel differentiated retransmission scheme for MPEG video streaming over wireless links," Int. J. Wireless and Mobile Computing, vol. 1, nos. 3/4, pp. 260-267, 2006.

[17] W. Wang, Z. Guo, X. Shen, and C. Chen, "Performance analysis of ARQ scheme in IEEE 802.16," in Proc. IEEE GLOBECOM'06, San Francisco, USA, Nov. 2006.

[18] D. Niyato and E. Hossain, "A queuing-theoretic and optimization-based model for radio resource management in IEEE 802.16 broadband wireless networks," IEEE Trans. Computers, vol. 55, no. 11, pp. 1473-1488, Nov.2006.

[19] Cayman Khalil, Mathieu Crussiere and Jean-Francis Helard," Cross Layer Resource allocation scheme under heterogeneous constraints for next generation high rate WPAN" International Journal of Computer Networks and Communications (IJCNC), vol.2, No.3, pp.152-168, May 2010.

[20] Mohammed El-Tashuni, Mohamed Hassan, Akram Bin Sediq," A Joint power allocation and adaptive channel coding scheme for image transmission over wireless channel" International Journal of Computer Networks and Communications (IJCNC), vol.2, No.3, May 2010. 


\section{Short Bio-graphy}

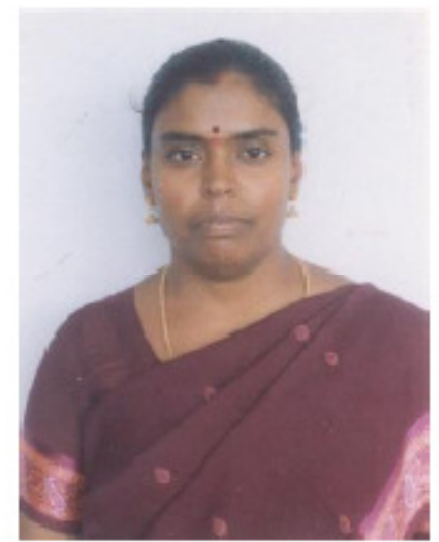

1 Mrs.G. Indumathi obtained Bachelor"s degree in Electronics and Communication Engineering and Master"s Degree in Communication Systems both from Madurai Kamaraj University, Madurai, India in 1990 and 1997 respectively. She is currently studying for a Ph.D degree at the department of Information and Communication Engineering, Anna University, Chennai. Her current research interests are in the areas of Wireless and Mobile communications and Digital signal Processing. She is a Life member in Indian Society for Technical Education(LM25836) and Fellow in Institution of Electronics and Telecommunications Engineers. She has published her research paper in the International Journal of Communication Networks and Information Security (IJCNIS).

2 Dr. Karuppasamy Murugesan obtained Bachelor "s degree in Electronics and Communication Engineering and Master"s Degree in Microwave and Optical Engineering both from Madurai Kamaraj University, Madurai , India in 1990 and 1993 respectively. He earned doctoral degree from Anna University, Chennai for his research work in the field of optical communications in the year 2001. He has also obtained post graduate diploma in Personnel Management and Industrial Relations from Annamalai University, Chidambaram, India in 1997.

He has published more than 30 papers in reputed National and International journals and conferences. His Research Interests include Optical Code Division Multiple Access, Optical Signal Processing Techniques, Lightwave Communication Systems, Optical Coding Theory and Ultrashort Light Pulse Communications. He is a Fellow in Institution of Electronics and Telecommunications Engineers, Life member in Indian Society for Technical Education and Associate Member in Institution of Engineers (India). He received „Best ISTE Chapter Secretary Award for Engineering Colleges" from ISTE (TN \& P Section) for the year 2007. He has more than 16 years of teaching experience in addition to one year of industrial exposure. Currently, $\mathrm{He}$ serves as the principal in Bharathiyar Institute of Engineering for Women, Attur in Tamilnadu, India. 